\title{
Prevalence and patterns of neurological involvement in Behcet's disease: a prospective study from Iraq
}

\author{
A Al-Araji, K Sharquie, Z Al-Rawi
}

J Neurol Neurosurg Psychiatry 2003;74:608-613

\begin{abstract}
Objectives: To determine the prevalence of neurological involvement in Behcet's disease in a prospective study, and to describe the clinical patterns of neurological presentation in this disease in patients attending a multidisciplinary clinic in Baghdad.

Methods: All patients attending the clinic who fulfilled the international study group criteria for the diagnosis of Behcet's disease were studied during a two year period starting in April 1999. Patients were assessed neurologically by a neuro-Behcetologist. All those with clinical neurological manifestations were sent for CSF examination, cranial magnetic resonance imaging, and magnetic resonance venography and were followed up to explore the patterns of neurological relapse.

Results: 140 patients with Behcet's disease were studied. Their mean age was 34.2 years (range 16 to 66$) ; 105(75 \%)$ were men and $35(25 \%)$ were women. The mean duration of the disease was 4.2 years (range 0.4 to 26 ). Twenty patients (14\%) had neurological involvement (neuro-Behcet's disease); 14 of these $(70 \%)$ were men and six $(30 \%)$ women. The mean age at the first neurological presentation was 34.1 years. The mean duration of follow up of patients with neuro-Behcet's disease was 20.7 months. Ten patients with neuro-Behcet's disease $(50 \%)$ presented with parenchymal CNS involvement, six $(30 \%)$ with intracranial hypertension, and four $(20 \%)$ with a mixed pattern of both parenchymal CNS involvement and intracranial hypertension.

Conclusions: Careful neurological assessment of patients with Behcet's disease may show a relatively high prevalence of neuro-Behcet features, and though the clinical patterns of presentation are characteristic a mixed pattern may occur.
\end{abstract}

See end of article for authors' affiliations

Correspondence to: Dr Adnan H Al-Araji, PO Box 28595, Code 12631, Baghdad, Iraq ahalaraji@uruklink.net

Received

17 October 2002

In revised form

24 December 2002

Accepted

17 January 2003

B ehcet's disease is a chronic relapsing multisystem disease of unknown aetiology. ${ }^{1}$ It bears the name of a Turkish dermatologist, Hulusi Behcet, who first described the disease in $1937 .^{2}$ Vasculitis is believed to be the underlying pathological process. ${ }^{3}$

The disease commonly affects young adults and can involve the skin and mucous membranes, eyes, joints, vascular system, lungs, gastrointestinal tract, and nervous system. ${ }^{4}$ As there are no definite diagnostic tests for Behcet's disease, the diagnosis relies on recognition of its various clinical features. These are present in different combinations, depending on the individual patient and the country of origin.

The most widely accepted diagnostic criteria are those of the international study group (ISG). ${ }^{5}$ These require the presence of recurrent oral ulcers plus two of the following: recurrent genital ulcerations, typical defined eye lesions, typical defined skin lesions, or a positive pathergy test (a skin hypersensitivity reaction to a non-specific physical insult; when positive, the response consists of a papule or pustule that develops after 24 to 48 hours at the site of a needle prick to the $\operatorname{skin}^{6}$ ).

It is recognised that Behcet's disease has a peculiar epidemiology; it is prevalent around the Mediterranean basin, especially in its eastern part, and in the eastern rim of Asia, but less so in other parts of the world. Geographical and ethnic variations in disease expression and severity are reported.$^{8}$ Iraq, which has a population of more than 22 million, is a Middle Eastern country with a relatively high prevalence of the disease. ${ }^{1011}$

Neurological involvement is well described in Behcet's disease (neuro-Behcet's disease), with variable prevalence rates of between $2.2 \%$ and $50 \%{ }^{8}{ }^{12-19}$ The prevalence is affected by many factors, including the interest and specialty of the unit from which patients were collected, the duration of follow up of the disease, the type of study (whether retrospective or pro- spective), regional and ethnic variations in disease expression, and the care with which the neurological assessment is done.

Different classifications of the patterns of neurological presentation are described, ${ }^{20-23}$ including a classification from our own neurology unit based on retrospective data. ${ }^{24}$

In this prospective study we aimed to determine the prevalence of neurological involvement and the clinical patterns of neurological presentation in patients attending a multidisciplinary Behcet's disease clinic in Baghdad, Iraq.

\section{METHODS}

In April 1999, a new Behcet's disease clinic was established at Baghdad Teaching Hospital. This is a multidisciplinary clinic to which referrals come from various specialties in different parts of the country. It is the only such specialised clinic in the country. This survey was designed to study Behcet's disease patients attending the clinic over a two year period, starting in April 1999 and ending in March 2001. It included all patients attending the clinic who fulfilled the ISG criteria for the diagnosis of Behcet's disease ${ }^{5}$ at the first visit or during the follow up period.

The following tests were carried out in all patients: complete blood count, erythrocyte sedimentation rate, urine analysis, and measurements of $\mathrm{C}$ reactive protein, rheumatoid factor, and antinuclear antibody. Other laboratory tests and radiological examinations were done as indicated clinically. The pathergy test was done by pricking the flexor aspect of forearm with a blunt gauge 20 needle and reading the result 48 hours later.

The patients were assessed by a dermatologist, a rheumatologist, an ophthalmologist, and other specialists as indicated. Every possible effort was made to rule out conditions that may simulate Behcet's disease. 
All the patients were assessed neurologically by one of the authors (AHA). A careful history was taken and a full neurological examination was done. In patients whose neurological complaints occurred before the first visit to the clinic, previous records and investigations were reviewed thoroughly. Patients with neurological findings suggestive of involvement of the nervous system by the disease were regarded as cases of neuro-Behcet's disease, while patients who complained of headache only, with no other symptoms or signs of neurological involvement, were not considered to have neuro-Behcet's disease.

Nineteen of the 20 patients with neuro-Behcet's disease had an examination of their cerebrospinal fluid (CSF), and this was repeated as indicated clinically during the follow up period.

Cranial magnetic resonance imaging (MRI) and magnetic resonance venography (MRV) were done in all patients with neuro-Behcet's disease, using a Philips Gyroscan NT (3000) superconducting 1.5 tesla instrument, with $23 \mathrm{mT}$ gradient power (Philips Inc, Einthoven, Netherlands). Axial T1 and T2 weighted images, coronal FLAIR sequences, MRV, and postcontrast $\mathrm{Tl}$ image sequences were undertaken. The examination was repeated when indicated clinically.

Follow up of neuro-Behcet's disease patients was done by the same neurologist, looking for patterns of neurological relapse.

\section{RESULTS}

In all, 140 patients with Behcet's disease were seen at the clinic during the two year period of the study. Their mean age at the time of assessment was 34.2 years (range 16 to 66). There were 105 men (75\%) and 35 women (25\%). The mean duration of the disease was 4.2 years (range 0.4 to 26). Eighteen patients $(13 \%)$ had had the disease for less than 12 months, 13 (9\%) for between 12 and 23 months, 31 (22\%) for between two and five years, and $78(56 \%)$ for more than five years. There were 134 Arab patients (96\%) and only six were Kurdish (4\%). Fifteen patients (10.7\%) had a positive family history of Behcet's disease.

Twenty patients (14.3\%) had neurological involvement; 14 of these $(70 \%)$ were men and six $(30 \%)$ women. Nineteen were Arabs and only one was Kurdish.

The mean age at the first neurological presentation was 34.1 years (range 21 to 52 ) and the mean duration of Behcet's disease at the first neurological presentation was 3.25 years (range $[-1]$ to 12 years). Two patients had their neurological presentation as the first manifestation of Behcet's disease, the other features appearing later. Three patients had their neurological presentation with the onset of the other features, while in the majority ( 15 patients) the neurological presentation was after the onset of the other manifestations of Behcet's disease. The mean duration of follow up of the patients with neuro-Behcet's disease was 20.7 months (range 6 to 33).

We categorised the neurological patterns of presentation into two main types, depending on the clinical neurological features and supported by CSF and MRI findings (table 1). Some patients had features of both types (mixed pattern).

\section{Parenchymal CNS pattern}

The first and the most common pattern (occurring in half the cohort) was the "parenchymal CNS" pattern, which included patients whose clinical features were suggestive of focal or diffuse parenchymal CNS involvement with no features to suggest intracranial hypertension. On CSF examination there was a normal opening pressure, usually with pleocytosis and/or a high protein; MRI commonly showed hyperintense T2 weighted lesions (plaques) while brain MRV showed no abnormalities. This pattern included well defined clinical forms (brain stem syndrome, cerebral stroke-like and psychiatric features) and a diffuse form suggestive of widespread
Table 1 Patterns of first neurological presentation in 20 patients with neuro-Behcet's disease

\begin{tabular}{ll}
\hline Pattern & No of patients (\%) \\
\hline 1. Parenchymal CNS & $10(50)$ \\
(a) Brain stem syndrome & 4 \\
(b) Cerebral stroke-like & 1 \\
(c) Psychiatric & 1 \\
(d) Diffuse & 2 \\
(e) Asymptomatic (silent) & 2 \\
2. Intracranial hypertension & $6(30)$ \\
3. Mixed & $4(20)$ \\
\hline
\end{tabular}

parenchymal CNS involvement, as described previously. ${ }^{24}$ The characteristic features of these forms were as follows:

- Brain stem syndrome (four patients): characterised by sudden or acute onset, taking days to reach the peak deficit. The findings were commonly a bilateral combination of cranial nerve, cerebellar, or pyramidal signs. Gradual improvement occurs in the course of the disease.

- Cerebral stroke-like (one patient): acute onset of unilateral neurological findings highly suggestive of a stroke, with features suggesting cerebral involvement, for example seizures.

- Psychiatric form (one patient): predominantly psychotic features.

- Diffuse form (two patients): acute or subacute onset with pseudobulbar signs including emotional lability, spastic dysarthria, swallowing difficulty, and brisk facial reflexes and jaw jerk; in these cases other features of parenchymal CNS involvement were reported, including pyramidal signs, a spinal sensory level, sphincter disturbances, and cerebellar signs.

There were two asymptomatic patients who had clear neurological signs (unilateral pyramidal signs) without a history of neurological complaints. These were relatively young ( 26 and 31 years) and had no risk factors for cerebrovascular disease or history of head trauma or perinatal birth injury. There was no pure spinal cord involvement in this series.

\section{Intracranial hypertension}

The other main pattern was "intracranial hypertension" which was manifested by headache, vomiting, and bilateral papilloedema, with no clinical or neuroradiological evidence of parenchymal CNS involvement. The CSF opening pressure was always increased, commonly with normal cells and protein. Cranial MRI was basically normal, with a normal ventricular system. MRV confirmed cerebral venous thrombosis in five of the six patients.

\section{Mixed pattern}

The other four patients had a mixed pattern: they showed clinical evidences of parenchymal CNS involvement (two with brain stem syndrome, one with the diffuse form, and one with the cerebral stroke-like form) in addition to features of intracranial hypertension (headache, vomiting, and bilateral papilloedema). The CSF opening pressure was increased and the MRI and MRV showed plaques and cerebral venous thrombosis in all four patients.

\section{Other clinical features}

None of the patients in this series had a pure meningeal pattern and none had peripheral nervous system involvement (peripheral neuropathy or myopathy).

Neurological relapses commonly took the same pattern as the initial presentations. Four relapses in two patients with the parenchymal CNS pattern and three relapses in two patients 
Table 2 Cumulative frequency of all neurological signs and symptoms in 20 patients with neuro-Behcet's disease

\begin{tabular}{ll}
\hline Neurological signs and symptoms & No of patients (\%) \\
\hline Pyramidal signs and symptoms & $14(70)$ \\
Headache & $12(60)$ \\
Papilloedema & $11(55)$ \\
Cranial nerves (excluding the optic nerve) & $10(50)$ \\
Cerebellar signs & $6(30)$ \\
Sphincter disturbances & $4(20)$ \\
Pseudobulbar signs & $3(15)$ \\
Meningeal signs & $3(15)$ \\
Sensory symptoms and signs & $3(15)$ \\
Personality changes & $1(5)$ \\
Epileptic fits & $1(5)$ \\
\hline
\end{tabular}

Table 3 Non-neurological manifestations in 20 patients with neuro-Behcet's disease

\begin{tabular}{lllll}
\hline & \multicolumn{2}{l}{$\begin{array}{l}\text { Presenting } \\
\text { feature }\end{array}$} & \multicolumn{2}{c}{$\begin{array}{l}\text { Cumulative } \\
\text { frequency }\end{array}$} \\
\cline { 2 - 5 } Clinical feature & $\mathrm{n}$ & $\%$ & $\mathrm{n}$ & $\%$ \\
\hline Oral ulcer & 8 & 40 & 20 & 100 \\
Genital ulcer & 3 & 15 & 14 & 70 \\
Skin lesions & - & - & 12 & 60 \\
Ocular lesions & 2 & 10 & 8 & 40 \\
Rheumatological & 3 & 15 & 8 & 40 \\
Vascular lesion & 2 & 10 & 5 & 25 \\
\hline
\end{tabular}

with intracranial hypertension had the same patterns as the initial presentations. However, one patient who presented initially with a parenchymal CNS pattern developed a relapse suggestive of a mixed pattern of both intracranial hypertension and parenchymal CNS involvement.

Pyramidal symptoms and signs were the most common features in the cumulative list of symptoms and signs throughout the course of the illness, as shown in table 2.

The non-neurological manifestations occurring during the course of the disease are shown in table 3. Ocular involvement in the form of anterior or posterior uveitis and retinal vasculitis was reported in eight patients with neuro-Behcet's disease $(40 \%)$. In the whole cohort of Behcet's disease patients, ocular involvement was reported in $42 \%$. The pathergy test was positive in 10 of the 15 patients in whom it was done.

\section{Investigations}

CSF examination was done in 19 patients. In those presenting with the parenchymal CNS pattern, 12 examinations were done in nine patients. In this group the CSF opening pressure was within normal limits, but six of the 12 CSF samples were abnormal: four had pleocytosis with or without raised protein, and two had raised protein only; the pleocytosis was lymphocytic in two (15 and 65 cells $/ \mathrm{mm}^{3}$ ) and mainly neutrophilic in the other two ( 15 and 45 cells $/ \mathrm{mm}^{3}$ ). The sugar content was normal in all samples.

The six patients who presented with intracranial hypertension all had increased CSF opening pressure (more than $200 \mathrm{~mm} \mathrm{CSF}$ )on at least one occasion; otherwise their CSF constituents were normal except for one sample which had a raised protein level.

In patients presenting with the mixed pattern, the CSF opening pressures were increased on at least one occasion in each case, and the CSF findings were abnormal in two. The abnormalities included pleocytosis (15 and 32 lymphocytes/ $\mathrm{mm}^{3}$ ) in both, while one patient had a raised protein (92

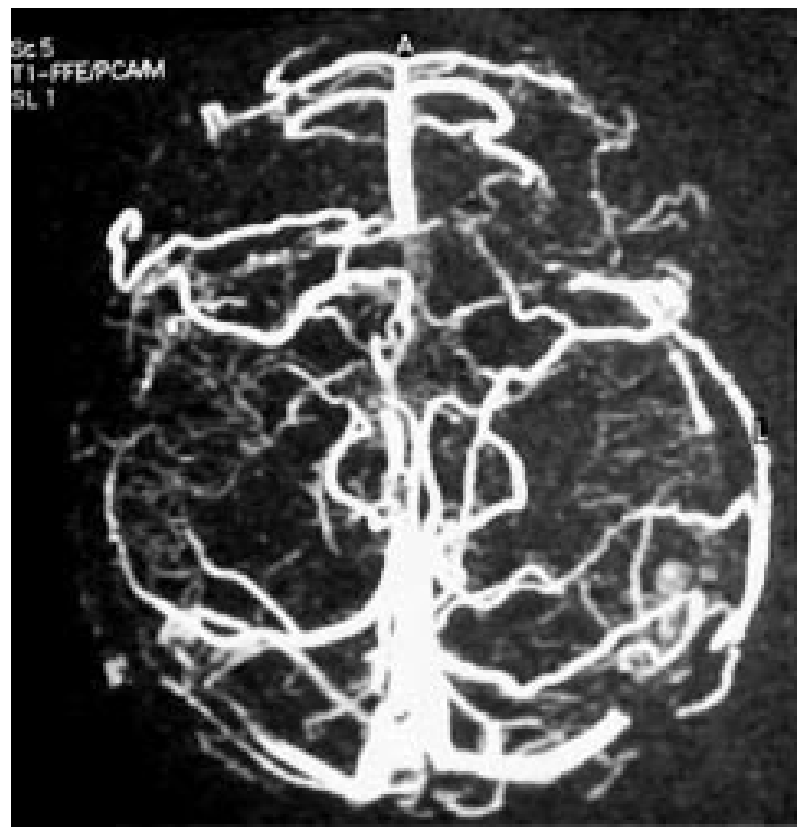

Figure 1 Brain magnetic resonance venography showing obliteration of the mid portion of the superior saggital sinus in a patient with Behcet's disease who present with intracranial hypertension.

$\mathrm{mg} / \mathrm{dl})$. CSF protein electrophoresis for oligoclonal IgG bands was not available during the study period.

Cranial MRI and MRV were done in all 20 patients with neuro-Behcet's disease on at least one occasion. These showed single or scattered lesion (plaques) in eight of the 10 patients presenting with the parenchymal CNS pattern, with normal MRV. Cranial MRI was normal in all six patients presenting with the intracranial hypertension pattern, and cranial MRV showed cerebral venous thrombosis in five of these (fig l). In patients presenting with the mixed pattern, cranial MRI and MRV showed both plaques and cerebral venous thrombosis in all four cases. The plaques that were seen in 12 patients (eight in the parenchymal CNS pattern and four in the mixed pattern) were recognised as hyperintense parenchymal lesions on T2 weighted images (figs 2 and 3 ) and as bright focal lesions on FLAIR sequences, except in one patient in whom one of the plaques was not visualised by FLAIR but was clearly seen after gadolinium enhancement as an enhanced focal parenchymal lesion. The Tl appearance of the plaques was variable: in the majority (seven of the 12 patients), the lesions appeared as hypointense focal lesions; in two patients, plaques were not visualised (isointense); in two patients, there were mixed patterns of hypointense and isointense lesions; and only one patient showed a hyperintense Tl weighted image focal lesion surrounded by a dark rim, suggestive of haemorrhagic plaque.

The distribution of the plaques was as follows: cerebral white matter in 11 patients (92\%), brain stem in five (42\%), thalamus and basal ganglia in four (33\%), and cortical grey matter in one $(8 \%)$. A periventricular distribution of the plaques, as is classically seen in multiple sclerosis, was not observed.

In the two patients with parenchymal CNS presentation and normal MRI, the intervals between the onset of the clinical presentation and the MRI study were two and six weeks.

Follow up MRI in two patients with parenchymal CNS involvement who had plaques at the initial assessment showed a reduction in the number and size of the plaques in one patient after three months from the time of the initial assessment and in the other patient after six months. 


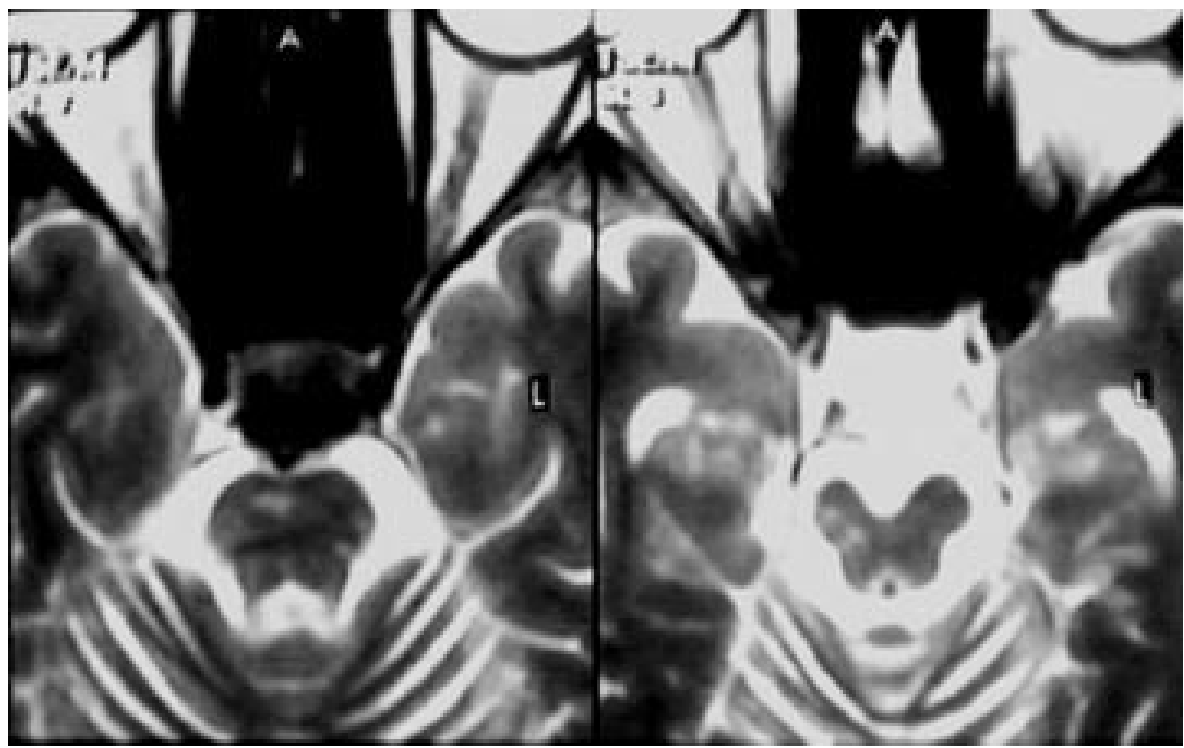

Figure 2 Brain magnetic resonance imaging: T2 weighted image showing hyperintense multiple plaques within the pons and the right side of the midbrain in a patient with Behcet's disease who present with parenchymal CNS involvement.

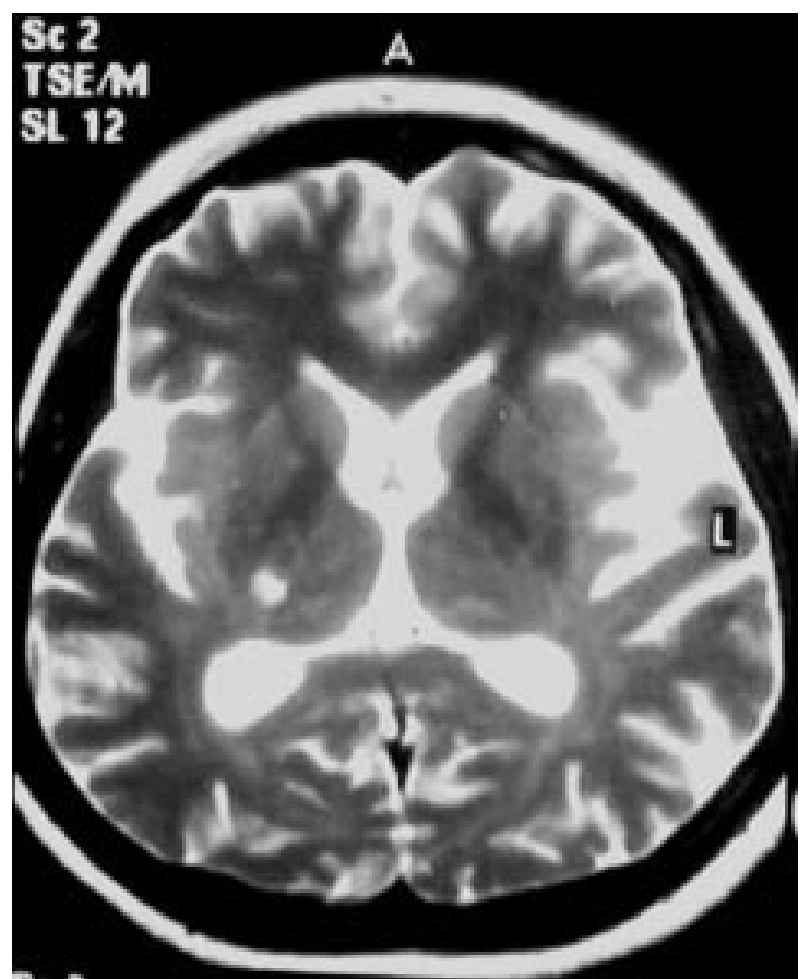

Figure 3 The same patient as in fig 2 showing hyperintense T2 weighted image of lesion within the posterior limb of the right internal capsule.

\section{DISCUSSION}

There have been many reports describing the prevalence rates for the neurological involvement in Behcet's disease from different countries. ${ }^{8}{ }^{12-19}$ These were mainly retrospective, from different units with variable interest in the disease. The present study was designed to investigate this issue prospectively over a two year period in a multidisciplinary clinic. The initial neurological assessment and the follow up were done by a neurologist with an interest in neuro-Behcet's disease.

The prevalence of neuro-Behcet's disease among our patients was $14.3 \%$, which is higher than the $5.3 \%$ in the only similar prospective study that we could trace (from Istanbul in $\left.1989^{14}\right)$. That study was carried out over a one year period and included 323 patients with Behcet's disease attending a Behcet's disease clinic. Their initial neurological assessment was done by a rheumatologist at the Behcet's clinic before referal to the neurology department, which might explain the difference in prevalence. Another possible explanation is the well known regional and ethnic variation in disease expression in Behcet's disease. ${ }^{89}$ MRI was not available at that time. It has been observed that the CNS is involved in a large proportion of patients with Behcet's disease in the Arabian region. ${ }^{21}$

Even in a multidisciplinary clinic, referrals may be biased toward the specialty of the most interested and active specialists attached to the clinic. This might have an effect on the reported prevalence rates for the different manifestations of the disease. In our Behcet's disease clinic, we try to reduce this bias to a minimum by encouraging referrals from all subspecialties and by encouraging patients to refer themselves to the clinic.

The results of the current study represent the prevalence of neurological involvement in Behcet's disease from a hospital based clinic and not in the community. Community based studies generally include milder disease manifestations. ${ }^{25}$ Carefully planned community based studies in different countries are needed to determine the prevalence of neurological involvement in Behcet's disease, as neurological manifestations represent a major cause of morbidity and mortality of this disease. ${ }^{26}$

We categorised the patterns of neurological presentation into two main types depending on the clinical findings, supported by the CSF results and cranial MRI and MRV: a parenchymal CNS pattern and an intracranial hypertension pattern. This classification has clinical, aetiopathological, therapeutic, and prognostic implications, as described in detail previously. ${ }^{24}$ It is a modification of our previously reported classification, which was based on retrospective data and lacked the benefit of MRI, which was not available at the time. The major modification is the finding of a "mixed pattern," which will be discussed below. Most of the cases of neuro-Behcet's disease reported to date can be grouped into this simple and practical classification. This will enable the practising clinician to remember the possibility of Behcet's disease when faced with the patterns and forms described in the classification. It does not, of course, cover every single rare and unusual manifestation of neuro-Behcet's disease, but we 
believe this shortcoming is not a major issue and it does not invalidate its usefulness.

The parenchymal CNS pattern was the most common form of presentation in our series, as well as in most of the large series of neuro-Behcet's disease so far reported. ${ }^{14} 18$ 21-24 26 This pattern is subdivided into various forms that represent commonly encountered neurological entities easily recognised on careful neurological evaluation. The main differential diagnosis of this pattern is multiple sclerosis. Internuclear ophthalmoplegia, gaze palsy, and optic neuritis are common clinical findings in multiple sclerosis, but these were not seen in our cases of neuro-Behcet's disease. Spinal cord involvement is common in multiple sclerosis but not in neuro-Behcet's disease. The systemic features of Behcet's disease are useful in directing attention to this disorder, and the presence of CSF pleocytosis is another clue suggestive of neuro-Behcet's disease. Cranial MRI is also very useful, the characteristic periventricular distribution in multiple sclerosis being uncommon in neuro-Behcet's disease. There is a detailed discussion of the differential diagnosis of the various forms of the parenchymal CNS pattern in our previous paper on neuro-Behcet's disease. ${ }^{24}$

There were two asymptomatic ( silent) patients who did not complain of neurological symptoms but who had definite neurological signs. There were no reasonable explanations for their neurological findings other than Behcet's disease. This should encourage a careful neurological assessment in all patients with Behcet's disease, even if they are asymptomatic from a neurological viewpoint. This might prevent missed or delayed diagnoses of neuro-Behcet's disease, which may have serious consequences.

The intracranial hypertension pattern of presentation is more often reported from the Middle East $^{121627-30}$ and France, ${ }^{31}$ and less often from Japan and other parts of the world. ${ }^{22}{ }^{32}$ In this study, about one third of the patients with neuro-Behcet's disease had this pattern, but if we combine it with the mixed pattern and with the follow up data, then more than half the patients had this type of presentation-a greater prevalence than has been reported from other countries. Geographical and ethnic variations in disease expression could be a reason for this. Another reason might be the careful neurological assessment and the routine use of MRV in our current study. The main differential diagnosis of this pattern-after exclusion of an intracranial mass by proper neuroimaging - is idiopathic intracranial hypertension. The predominantly male sex of the patients, the systemic features of Behcet's disease, and the common finding of cerebral venous thrombosis on brain MRV are features that direct the attention to neuro-Behcet's disease.

Our finding that one fifth of the patients with neuroBehcet's disease had a mixed pattern of both parenchymal CNS involvement and intracranial hypertension is interesting. It is generally believed that the underlying aetiopathology in parenchymal CNS involvement is small vessel vasculitis, while in intracranial hypertension it is cerebral venous thrombosis secondary to a haemostatic abnormality or small vessel vasculitis of the large cerebral veins or sinuses. ${ }^{24}$ Many believe that these two main presentations represent different aetiopathological processes and thus that the neurological manifestations of Behcet's disease should be divided into "neuroBehcet's syndrome" (NBS; the parenchymal CNS involvement) and "vasculo-Behcet," representing cases with cerebral venous thrombosis. ${ }^{18} 2311$ A mixture of both presentations in the same patient has not been well described before. Our findings suggest either that both patterns of presentation are caused by small vessel vasculitis involving the CNS parenchyma and the large cerebral veins or sinuses, or, if these are different aetiopathological processes, that they can coexist in the same patient. We prefer not to separate the neurological manifestations of Behcet's disease into the so called NBS and vasculo-Behcet types, but to group them together and use the term neuro-Behcet's disease for both-as both may result from the same aetiopathological processes, as suggested in this study, and because both represent direct involvement of the nervous system by Behcet's disease. As these neurological manifestations are usually dealt with and followed up at neurological clinics, the use of separate terms may be confusing and serves no useful practical purpose.

There were no cases with pure meningeal involvement in this series, and none of the patients had peripheral nervous system involvement. These presentations, though they have been reported, ${ }^{21} 2233$ are uncommon.

It is interesting to note that none of our patients had extrapyramidal features. These have only rarely been reported despite the common involvement of the basal ganglia on $\mathrm{MRI}^{34}$ and in histopathological series. ${ }^{35}$

Behcet's disease patients who complain of headache with no other neurological signs and symptoms to suggest neurological involvement were not regarded as having neuroBehcet's disease. These were mainly patients with primary headache syndromes (commonly migraine and tension-type headache). These types of headache are known to be prevalent in Behcet's disease, but it is generally believed that they are not related to a direct involvement of the nervous system by the disorder. $^{1822} 23$ A prospective study is needed to follow such patients and to determine the outcome.

Neurological relapses commonly took the same pattern as the presentation, but less commonly a different pattern may occur with relapse, as found in other surveys. ${ }^{1822-24}$

The onset of the neurological presentation commonly follows or coincides with the other systemic features of Behcet's disease. It is always preferable to ask routinely about these features in neurological patients from areas where Behcet's disease is prevalent. The neurological presentation may precede the other systemic features of Behcet's disease; as noted in the present study and in other reports, ${ }^{22-24}$ the pattern of the presentation, the ethnic origin, the CSF findings, the MRI and MRV findings, and the course of the disease may suggest the diagnosis.

Signs of motor dysfunction, as manifested by the pyramidal signs and symptoms, were the most common cumulative neurological findings during the course of the illness, in agreement with previous reports. ${ }^{142-24}$ These are the main disabling features of the parenchymal CNS presentation, while the impact on vision is the main danger for those who present with intracranial hypertension. ${ }^{24}$

Cranial MRI and MRV were very helpful in categorising the neurological involvement. Both should be used routinely in cases of suspected neuro-Behcet's disease, especially MRV, which may direct the attention to the possibility of cerebral venous thrombosis requiring anticoagulation. It may also reduce the need for more invasive neuroimaging in suspected cases of cerebral venous thrombosis.

\section{Conclusions}

The prevalence of neurological involvement is relatively high (14.3\%) in Iraqi patients with Behcet's disease attending a Behcet's disease clinic in Baghdad, and though the types of neurological presentation can be grouped into two main distinct patterns (parenchymal CNS and intracranial hypertension), a mixed pattern may also occur.

\section{ACKNOWLEDGEMENTS}

We thank Prof H Yazici, Department of Rheumatology, Cerrahpsa Medical Faculty, Istanbul, and Dr N Al-Ward, Department of Community Medicine, College of Medicine, Baghdad, for reviewing the manuscript.

\section{Authors' affiliations}

A Al-Araji, Behcet's Disease Clinic, Baghdad Teaching Hospital and Department of Neurology, College of Medicine, University of Baghdad, Iraq 
K Sharquie, Behcet's Disease Clinic, Baghdad Teaching Hospital and Department of Dermatology

Z Al-Rawi, Behcet's Disease Clinic, Baghdad Teaching Hospital, and Department of Rheumatology

Competing interests: none declared

\section{REFERENCES}

1 Yazici H, Yurdakul S, Hamuryudan V. Behcet's syndrome. Curr Opin Rheumatol 1999;11:53-7.

2 Behcet H. Uber rezidivierende Aphthose durch ein Virus-veursachtr Geschwur am Mund, am Auge und an den Genitalien. Dermatol Monatsschr 1937;36:1 152-7.

3 Ehrlich GE. Vasculitis in Behcet's disease [review]. Int Rev Immunol 1997; 14:81-8.

4 Yazici H, Yurdakul S, Hamuryudan V. Behcet's syndrome. In: Maddison PJ, Isenberg DA, Woo P, et al, eds. Oxford textbook of rheumatology, 2nd ed. Oxford: Oxford University Press, 1998:1394-402.

5 International Study Group for Behcet's disease. Criteria for diagnosis of Behcet's disease. Lancet 1990;335:1078-80.

6 Chang HK, Cheon KS. The clinical significance of a pathergy reaction in patients with Behcet's disease. J Korean Med Sci 2002;1 7:271-4.

7 O'Duffy JD. Behcet's syndrome. N Engl J Med 1990;322:326-7.

8 Gurler A, Boyvat A, Tursen U. Clinical manifestations of Behcet's disease. An analysis of 2147 patients. Yonsei Med J 1997:38:423-7.

9 Zouboulis CC, Kotter I, Diawari D, et al. Current epidemiological data from the German registry of Adamantiades-Behcet's disease. The 10th International Conference on Behcet's disease (programme and abstracts), 27-29 June. Berlin, 2002:57.

10 Sharquie K, Al-Araji A, Al-Rawi Z, et al. Behcet's disease in Iraqi patients. A prospective study from a newly established multidiscipline Behcet's disease clinic. In: Bang D, Lee E-S, Lee S, eds. Proceedings of the 9th international conference on Behcet's disease, 27-29 May. Seoul, 2000:60-3.

11 Al-Rawi Z, Neda AH. Prevalence of Behcet's disease among Iraqis. The 10th International Conference on Behcet's disease (programme and abstracts), 27-29 June. Berlin: 2002:71.

12 Fadli ME, Youssef MM. Neuro-Behcet syndrome in the United Arab Republic. Eur Neurol 1973;9:76-89.

13 Shimizu T, Ehrlich GE, Inaba G, et al. Behcet's disease (Behcet syndrome). Semin Arthritis Rheum 1979;8:223-60.

14 Serdaroglu P, Yazici H, Ozdemir C, et al. Neurological involvement in Behcet syndrome - a prospective study. Arch Neurol 1989;46:265-9.

15 Benamour S, Zeroual B, Bennis R, et al. Maladie de Behcet: 316 cas. Press Med 1990;19:1485-9.
16 Al-Dalaan AN, Al-Balaa SR, El-Ramahi K, et al. Behcet disease in Saudi Arabia. J Rheumatol 1994;21:658-61.

17 Davitchi F, Shavran F, Akbarin M, et al. Behcet's disease: analysis of 3443 cases. APLAR J Rheumatol 1997;1:2-5

18 Siva A, Kantarci OH, Saip S, et al. Behcet's disease:diagnostic and prognostic aspects of neurological involvement. J Neurol 2001:248:95-103.

19 Ben Taarit C, Turki S, Benmaiz H. Neurological manifestations in Behcet's disease. Forty observations in a cohort of 300 patients. J Mal Vasc 2002;27:77-81

20 Pallis CA, Fudge BJ. The neurological complications of Behcet's syndrome. Arch Neurol Psychiatry 1956;75:1-4.

21 Al-Kawi MZ. Neuro-Behcet disease: a review. J Trop Geogr Neurol 1992;2:49-56

22 Kidd D, Steuer A, Denman AM, et al. Neurological complications in Behcet's syndrome. Brain 1999;122:2138-94.

23 Akman-Demir G, Serdaroglu P, Tasci B, for the Neuro-Behcet Study Group. Clinical patterns of neurological involvement in Behcet's disease:evaluation of 200 patients. Brain 1999;122:2171-81.

24 Al-Fahad S, Al-Araji A. Neuro-Behcet's disease in Iraq: a study of 40 patients. J Neurol Sci 1999;170:105-11.

25 Yardakul S, Gunaydin I, Tuzun Y. The prevalence of Behcet's syndrome in a rural area in northern Turkey. J Rheumatol 1988;15:820-2

26 Serdaroglu P. Behcet's disease and the nervous system [review]. J Neurol 1998;245: 197-205

27 Bashir R, Al-Kawi MZ, Jinkins JR, et al. Neuroimaging features of Behcet's disease. Ann Saudi Med 1989;9:11-15.

28 Daif A, Awada A, al-Rajeh S, et al. Cerebral venous thrombosis in adults. A study of 40 cases from Saudi Arabia. Stroke 1995;26:1 193-5.

29 Al-Araji A, Sharquie K, Al-Rawi Z. Intracranial hypertension. A characteristic neurological pattern of presentation of Behcet's disease in 10 Arab patients. In: Bang D, Lee E-S, Lee S, eds. Proceeding of the 9th international conference on Behcet's disease, 27-29 May. Seoul 2000:338-40.

30 el Bahri Ben Murad F, Fredj M, Skandarani L, et al. Cerebral venous thrombosis and Behcet's disease. Tunis Med 2002;80:87-9.

31 Wechsler B, Vidaihet $M$, Piette JC, et al. Cerebral venous thrombosis in Behcet's disease: clinical study and long-term follow-up of 25 cases. Neurology 1992;42:614-18.

32 Ikeda K, Abe M, Iwasaki Y, et al. Neuro-Behcet's disease in Japan. Neurology 1996:47:614-15.

33 Namer IJ, Karabudak R, Zileli T, et al. Peripheral nervous system involvement in Behcet's disease. Eur Neurol 1987;26:235-40.

34 Lee SH, Yoon PH, Park SJ, et al. MRI findings in neuro-Behcet's disease. Clin Radiol 2001;56:485-94.

35 Hadfield MG, Aydin F, Lippman HR, et al. Neuro-Behcet's disease. Clin Neuropathol 1996;15:249-55. 